\title{
Coherent Manipulation of Orbital Feshbach Molecules of Two-Electron Atoms
}

\author{
G. Cappellini, ${ }^{1,3,4, *}$ L. F. Livi, ${ }^{1,3,4}$ L. Franchi, ${ }^{1,4}$ D. Tusi, ${ }^{2}$ D. Benedicto Orenes, ${ }^{3}$ \\ M. Inguscio, ${ }^{1,2,3}$ J. Catani, ${ }^{3,2,4}$ and L. Fallani ${ }^{1,2,3,4}$ \\ ${ }^{1}$ Dipartimento di Fisica e Astronomia, Università degli Studi di Firenze, I-50019 Sesto Fiorentino, Italy \\ ${ }^{2}$ LENS European Laboratory for Nonlinear Spectroscopy, I-50019 Sesto Fiorentino, Italy \\ ${ }^{3}$ INO-CNR Istituto Nazionale di Ottica del CNR, \\ Sezione di Sesto Fiorentino, I-50019 Sesto Fiorentino, Italy \\ ${ }^{4}$ INFN Istituto Nazionale di Fisica Nucleare, Sezione di Firenze, I-50019 Sesto Fiorentino, Italy
}

(Received 23 October 2018; revised manuscript received 10 January 2019; published 11 February 2019)

Ultracold molecules have experienced increasing attention in recent years. Compared to ultracold atoms, they possess several unique properties that make them perfect candidates for the implementation of new quantum-technological applications in several fields, from quantum simulation to quantum sensing and metrology. In particular, ultracold molecules of two-electron atoms (such as strontium or ytterbium) also inherit the peculiar properties of these atomic species, above all, the possibility to access metastable electronic states via direct excitation on optical clock transitions with ultimate sensitivity and accuracy. We report on the production and coherent manipulation of molecular bound states of two fermionic ${ }^{173} \mathrm{Yb}$ atoms in different electronic (orbital) states ${ }^{1} S_{0}$ and ${ }^{3} P_{0}$ in the proximity of a scattering resonance involving atoms in different spin and electronic states, called orbital Feshbach resonance. We demonstrate that orbital molecules can be coherently photoassociated starting from a gas of ground-state atoms in a threedimensional optical lattice by observing several photoassociation and photodissociation cycles. We also show the possibility to coherently control the molecular internal state by using Raman-assisted transfer to swap the nuclear spin of one of the atoms forming the molecule, thus demonstrating a powerful manipulation and detection tool of these molecular bound states. Finally, by exploiting this peculiar detection technique we provide the first information on the lifetime of the molecular states in a many-body setting, paving the way towards future investigations of strongly interacting Fermi gases in a still unexplored regime.

DOI: 10.1103/PhysRevX.9.011028

\section{INTRODUCTION}

The experimental study of ultracold molecules gained significant momentum in the past decade, as molecules can considerably extend the range of application of ultracold quantum technology to a wealth of different phenomena and regimes hardly accessible to atoms [1]. For instance, simple molecules are a valuable resource for high-precision spectroscopy, as they can exhibit much increased sensitivities (with respect to atoms) to the high-precision measurements of fundamental constants (e.g., the electron-to-proton mass ratio) or to the search of new physics and elusive quantum effects [2,3]. From a many-body perspective, interactions between molecules show a significantly richer physics than

\section{*cappellini@lens.unifi.it}

Published by the American Physical Society under the terms of the Creative Commons Attribution 4.0 International license. Further distribution of this work must maintain attribution to the author(s) and the published article's title, journal citation, and DOI.
Subject Areas: Atomic and Molecular Physics

that of neutral atoms and great effort has been recently devoted to the realization of ultracold gases of polar molecules [4-10], eventually reaching the Fermi-degenerate regime [11]. The interest for such systems relies in the possibility of exploiting the strong dipole-dipole interaction connected with the permanent electric dipole of molecules, which could allow for the observation of new strongly correlated quantum phases $[12,13]$. Furthermore, the study of interactions between molecules and/or atoms prepared in well-controlled quantum states enabled the first experimental studies in the emerging field of ultracold quantum chemistry $[14,15]$.

While extraordinary progress has been made in very recent years in the cooling of room-temperature stable molecules [16-19], the controlled synthesis of molecular bound states starting from ensembles of ultracold atoms is still a leading direction of research. Most of the experimental activity in this context was focused on alkali atoms, where magnetic Feshbach resonances can be used to adjust the interaction between cold atoms and associate them in weakly bound dimers with tunable binding energy [20]. 
Extending this study to molecules made by alkalineearth atoms (or alkaline-earth-like atoms such as ytterbium) would open completely new scenarios. Indeed, the twoelectron structure of these atoms entails the existence of low-lying (spin-triplet) metastable electronic states that can be excited from the (spin-singlet) ground state on narrow intercombination optical transitions. Among these, the doubly forbidden transition connecting the ground state ${ }^{1} S_{0}$ and the excited state ${ }^{3} P_{0}$ (with sub- $\mathrm{Hz}$ natural linewidth) is well known in the context of frequency metrology, as it is used for realizing the most precise and accurate atomic clocks to date at the $10^{-18}$ level [21-23]. The coherent manipulation of molecular states made by twoelectron atoms could disclose new opportunities in this context, with the possible development of sub-Hz-linewidth molecular atomic clocks with enhanced "sensing" capabilities [24]. Previous experimental work in a related context include the production and manipulation of cold $\mathrm{Sr}$ molecules on the $\left|{ }^{1} S_{0}\right\rangle \rightarrow\left|{ }^{3} P_{1}\right\rangle$ transition $[15,25,26]$ and early studies on the photoassociation of molecular bound states of $\mathrm{Yb}$ atoms in metastable states [27-29]. However, no coherent manipulation of molecules made by twoelectron atoms in clock states ${ }^{1} S_{0}-{ }^{3} P_{0}$ of metrological relevance has been reported yet.

The manipulation of those "clock" molecules is particularly favored in ${ }^{173} \mathrm{Yb}$, as the molecular levels can be controlled thanks to a peculiar kind of Feshbach resonance, called orbital Feshbach resonance (OFR) [30]. This resonance, based on the interorbital spin-exchange interaction mechanism arising in two-electron fermions with a clock (orbital) degree of freedom (d.o.f.) [31,32], was observed for the first time in Refs. $[29,33]$, thanks to the favorable scattering properties of ${ }^{173} \mathrm{Yb}$. Besides allowing for the possibility of tuning the binding energy of the ${ }^{1} S_{0}-{ }^{3} P_{0}$ molecular states, the OFR is predicted to exhibit very rich consequences for ultracold many-body physics. First, it is characterized by unusually small, yet experimentally accessible, energy scales, which may lead to the investigation of fermionic superfluidity in still-unexplored regimes [30, 34-37]. In addition, its combination with new techniques for the generation of robust single-photon spin-orbit coupling [38-40] could lead to the emergence of ultracold topological phases with Majorana zero-energy excitation modes [41-44]. Also for those applications, the demonstration of coherent optical manipulation of ${ }^{1} S_{0}-{ }^{3} P_{0}$ bound states is of utmost relevance.

In this paper, we report on the coherent production of weakly bound molecules made by ${ }^{1} S_{0}-{ }^{3} P_{0}{ }^{173} \mathrm{Yb}$ atom pairs in the proximity of the OFR and on the coherent optical manipulation of their internal state. Figure 1 shows a sketch of the system under investigation and of the main experimental tools that we use for the control of the molecular state. The paper is organized as follows. In Sec. II, we recall the main properties of the OFR in two-electron fermions. In Sec. III, we demonstrate the



FIG. 1. Sketch of the experimental system. Ultracold ${ }^{173} \mathrm{Yb}$ atoms are trapped in optical lattices (of variable dimensionality depending on the specific experiments). The electronic (orbital) state is manipulated by an ultranarrow clock laser, while a pair of Raman laser beams controls the nuclear-spin state. Interactions between atoms in different internal states are controlled with a magnetic field tuned in proximity of the orbital Feshbach resonance.

possibility of coherent photoassociation of molecules via the optical clock transition, showing high-fidelity photoassociation and photodissociation cycles. In Sec. IV, we show that, by exploiting the $\mathrm{SU}(\mathrm{N})$ character of the interactions in two-electron atoms, we can use coherent Raman coupling to control the nuclear-spin composition of the molecules as a powerful manipulation and detection tool. In Sec. V, we demonstrate the long lifetime of isolated molecules and provide first measurements on the lifetime of the molecular states in a many-body setting. Section VI is devoted to conclusions.

\section{ORBITAL FESHBACH RESONANCE IN ${ }^{173}$ Yb}

In this section, we briefly review the properties of the orbital Feshbach resonance exhibited by ${ }^{173} \mathrm{Yb}$ atoms (see Refs. [29,30,33] for more details). This scattering resonance arises from the existence of two different d.o.f., an electronic (orbital) state and a nuclear-spin state, which are coupled by an interorbital spin-exchange interaction [31,32]. We consider two atoms in two different orbital states $|g\rangle={ }^{1} S_{0}$ and $|e\rangle={ }^{3} P_{0}$, and two different nuclearspin projection states $|\uparrow\rangle$ and $|\downarrow\rangle$ chosen arbitrarily out of the $I=5 / 2$ nuclear-spin manifold. Atom-atom interactions are described by two different short-range scattering potentials corresponding to the two exchange-symmetrized two-body states $\left|e g^{ \pm}\right\rangle=|g \uparrow, e \downarrow\rangle \mp|g \downarrow, e \uparrow\rangle$. A nonzero magnetic field introduces a coupling between the $\left|e g^{ \pm}\right\rangle$ 
states and defines the long-distance asymptotic scattering channels $|c\rangle=|g \downarrow, e \uparrow\rangle$ (closed channel) and $|o\rangle=$ $|g \uparrow, e \downarrow\rangle$ (open channel). As in ordinary Feshbach resonances, the two-body $s$-wave scattering length diverges when the scattering state for two atoms colliding in the $|o\rangle$ channel becomes quasiresonant with a bound state of the $|c\rangle$ potential. In the specific case of ${ }^{173} \mathrm{Yb}$, the resonance occurs with the least bound state of $|c\rangle$, which has a binding energy of only a few kilohertz. This very small value allows the possibility of accessing the resonance regime even if the magnetic-field tuning is strongly suppressed by the purely nuclear character of the atomic spin (with a differential magnetic sensitivity of the two channels of $\approx 110 \mathrm{~Hz} / \mathrm{G} \times \Delta m$, where $\Delta m$ is the difference in the spin projection quantum numbers of the $|\uparrow\rangle$ and $|\downarrow\rangle$ states).

It is indeed because of the last property discussed above that the OFR exhibits the character of a narrow Feshbach resonance [37], while keeping a very broad tunability in terms of magnetic field accessibility. As an example, for ${ }^{173} \mathrm{Yb}$ atoms in the $|\uparrow\rangle=|+5 / 2\rangle,|\downarrow\rangle=|-5 / 2\rangle$ states, the resonance is located at $B_{0} \simeq 40 \mathrm{G}$, with a zero crossing of the scattering length at around $B \simeq 400 \mathrm{G}$. This unusual feature could lead to the first experimental investigation of the BEC-BCS crossover close to a narrow Feshbach resonance, allowing us to overcome the strict magneticfield stability requirements for ordinary Feshbach resonances in alkali gases [37]. Furthermore, the OFR is also characterized by an unusually small energy separation between the coupled scattering channels [34], lying in the same kilohertz range as the Fermi energy. This property results in intriguing many-body physics. For instance, it was suggested that the resulting many-body superfluid state has to be described in terms of two coupled order parameters [30], opening the door to study the physics of two-gap superconductors, with the prediction of new collective excitations and the emergence of the long-sought massive Leggett mode [35,36].

A natural direction for the experimental investigation of these phenomena is to follow the path of fermionic alkali gases, where the first experiments aimed at the formation of ultracold fermionic molecules on the BEC side of the resonance, followed by measurements of their stability [45] and by their Bose condensation in a superfluid state [46-48].

However, the same trait that makes the system interesting (the small energy scales) also precludes the possibility of a direct application of the experimental tools that were developed to control and detect Feshbach molecules of alkali atoms. For instance, already a direct imaging of the molecules is prohibited by their binding energy being much smaller than the linewidth of the imaging transition. Therefore, different tools need to be implemented (we discuss them in Sec. IV).

We conclude this section by mentioning that the OFR follows a very simple scaling relation with the magnetic

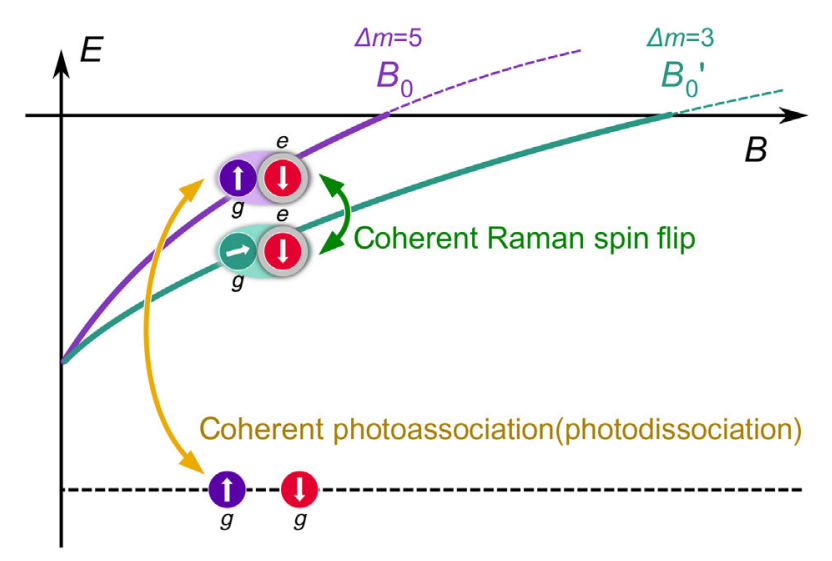

FIG. 2. Sketch of the processes considered in this work and corresponding energy levels. The solid lines show the binding energies for ${ }^{173} \mathrm{Yb}|e\rangle-|g\rangle$ atom pairs with different values of $\Delta m$ (difference between the nuclear-spin projection quantum numbers) and $B_{0}\left(B_{0}^{\prime}\right)$ denotes the position of the OFR center. The arrows show the different laser transitions used in the experiment for molecular production(and dissociation) and control of the internal state.

field $B$ and the difference in nuclear-spin projection quantum numbers $\Delta m$ of the interacting atoms. As a matter of fact, the scattering length $a$ is a universal function of $B \Delta m: a(B, \Delta m)=\tilde{a}(B \Delta m)$. This property stems from the $\mathrm{SU}(\mathrm{N})$ character of the interactions in two-electron fermions $[29,33,49]$, namely, the independence of the scattering potentials on the nuclear-spin state, which cause $\Delta m$ to affect only the Zeeman splitting between the two scattering channels (and not their individual properties). This behavior is exemplified by the binding energies on the BEC side of the resonance, which are sketched in Fig. 2 for two different atom pairs with $\Delta m=5$ and $\Delta m=3$ : while the two binding energies at zero field are degenerate, they differ at a finite magnetic field according to the same scaling $E_{b}(B, \Delta m)=\tilde{E}_{b}(B \Delta m)$. We take advantage of this scaling in the Raman-based spectroscopy discussed in Sec. IV.

\section{COHERENT ASSOCIATION OF ORBITAL MOLECULES}

In this section, we show how molecular bound states of ${ }^{173} \mathrm{Yb}$ atoms in different electronic states $(|g\rangle$ and $|e\rangle)$ can be produced by means of coherent photoassociation pulses of clock-laser light in a 3D optical lattice. In this configuration, the molecules are localized in individual lattice sites, preventing short-term inelastic losses and allowing for an easier study of their basic properties, as well as for the observation of long-lived coherent photoassociation and photodissociation cycles of orbital molecules. Moreover, the presence of the lattice confinement increases the stability of the dimer [50-52], allowing for the existence of bound states at magnetic-field values higher than the 
center of the free-space Feshbach resonance, located at approximately $40 \mathrm{G}[29,33]$, as well as for an increase of the low-field binding energy.

The experiment is initiated by preparing a Fermi gas of approximately $70 \times 10^{3}$ ytterbium atoms at a temperature $T=0.25 T_{F}$ (with $T_{F}$ the Fermi temperature) in the $m=$ $\pm 5 / 2$ nuclear-spin states. The gas is obtained by evaporating in a 3D optical trap at $1064 \mathrm{~nm}$ with final trapping frequency of $2 \pi \times(93,73,86) \mathrm{Hz}$ after having transferred all the atoms in the $m= \pm 5 / 2$ states by means of a combination of optical pumping pulses resonant with the nuclear-spin-selective $\left|{ }^{1} S_{0}\right\rangle \rightarrow\left|{ }^{3} P_{1}\right\rangle$ intercombination transition. A $3 \mathrm{D}$ optical lattice operating at the magic wavelength $\lambda_{L}=2 \pi / k_{L}=759 \mathrm{~nm}$ is adiabatically ramped up to a depth of $V_{0}=s E_{r}$, with $s=15$ and where $E_{r}=$ $\hbar^{2} k_{L}^{2} / 2 M$ is the recoil energy of the lattice, with $M$ being the atomic mass. The dipole trap at $1064 \mathrm{~nm}$ is then turned off with a 1-s-long linear ramp and the lattices are subsequently increased in $1 \mathrm{~ms}$ to a final lattice depth $s_{f}$ between 15 and 30 (sufficient to freeze the translational d.o.f.) depending on the specific experiment. This latticeloading procedure is adjusted to maximize the number of lattice sites occupied by a pair of $m= \pm 5 / 2$ atoms.

In order to produce orbital molecules we excite the $|g\rangle \rightarrow|e\rangle$ clock transition at a specific magnetic field $B$ by probing the atomic sample in the Lamb-Dicke regime with pulses of $\pi$-polarized clock-laser light generated by a frequency-doubled solid-state laser locked to an ultrastable optical cavity with a $<50 \mathrm{~Hz}$ linewidth [53], similarly to the experiment performed in Ref. [29]. In doubly occupied sites, different two-particle states can be excited at different clock-laser frequencies. While two-particle states of fermions with repulsive interactions have already been investigated both in $\mathrm{Yb}$ [31,32] and $\mathrm{Sr}$ [54] atoms, here we perform a detailed study of the molecular branch located in a lower-frequency region of the spectrum, which was previously observed in Ref. [29]. Figure 3 reports a series of typical clock spectra at $B=150 \mathrm{G}$ for different depths of the 3D lattice obtained by measuring the number of atoms in the excited $|e\rangle$ state after the clock-laser pulse. This is done by removing the residual ground-state atoms after the clock excitation with a resonant imaging pulse, after releasing the cloud from the lattice and pumping the $|e\rangle$ atoms into the ${ }^{3} D_{1}$ state with a $1389 \mathrm{~nm}$ laser. From there they then decay, through the intermediate ${ }^{3} P_{1}$ level, to the ground state, where they can be detected with the usual imaging procedure [55]. In the spectra of Fig. 3, the zerofrequency reference of the horizontal axis is the singleparticle $|g\rangle \rightarrow|e\rangle$ transition for individual atoms in the $m=-5 / 2$ state. This transition frequency depends only on the magnetic field and not on the lattice depth (residual light shifts induced by the lattice light are negligible at the resolution level of this experiment) and corresponds to the total energy of a pair of noninteracting $|e,-5 / 2\rangle$ and $|g, 5 / 2\rangle$ atoms, i.e., the threshold energy of the orbital

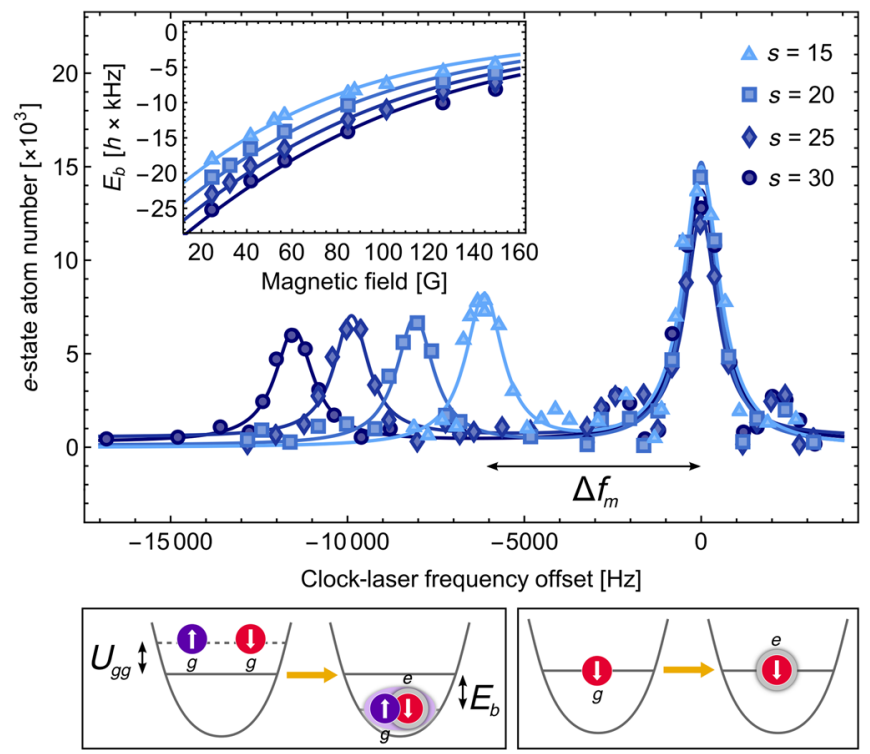

FIG. 3. Clock-laser spectroscopy of a $m= \pm 5 / 2$ sample of ${ }^{173} \mathrm{Yb}$ in a 3D lattice for different values of lattice depth and $150 \mathrm{G}$. The zero-frequency reference corresponds to the excitation of individual $m=-5 / 2$ atoms in singly occupied lattice sites, while the peaks at lower frequency correspond to the photoassociation process of $|g\rangle-|e\rangle$ bound states. Inset: Value of the binding energy as a function of the magnetic field for different lattice depths, and the solid lines are a fit with the theoretical model (see text for details).

Feshbach resonance. In every dataset, the peak located at lower frequency corresponds to the excitation of a $|g\rangle-|g\rangle$ pair to a $|g,+5 / 2 ; e,-5 / 2\rangle$ orbital molecular state characterized by a binding energy $E_{b}$. Since this photoassociation peak is selectively addressable, the detection of atoms in the $|e\rangle$ state at this excitation frequency is a reliable signal that molecules have been created. In the following, the notation $|g ; e\rangle$ could also be used for simplicity to indicate a generic molecular state of two atoms in $|g\rangle$ and $|e\rangle$ states. By measuring the distance $\Delta f_{m}$ between the single-particle peak and the molecular photoassociation peak, the binding energy can be determined as $E_{b}=$ $h \Delta f_{m}-U_{g g}$, where $h$ is the Planck constant and $U_{g g}$ is the repulsive Hubbard interaction energy of the two initial ground-state $m= \pm 5 / 2$ atoms calculated using the scattering length values reported in Ref. [56].

With this approach, we measured the binding energy $E_{b}$ of a $|g,+5 / 2 ; e,-5 / 2\rangle$ molecule as a function of the magnetic field $B$ for several values of the lattice depth ranging from $s_{f}=15$ to $s_{f}=30$. The results of these measurements are reported in the inset of Fig. 3. As anticipated before, it should be noted that a molecular state exists even at magnetic-field values higher than the position of the free-space resonance around $40 \mathrm{G}$ due to lattice confinement. The solid lines in the inset are a global fit of the full experimental dataset with the theoretical model discussed in Ref. [29] that derives the molecular 
binding energy by extending the problem of two atoms interacting in a harmonic trap with a single interaction channel [57] to the case of a singlet and triplet interaction channels coupled by a magnetic field. Anharmonic corrections due to the finite lattice depth have been evaluated numerically by diagonalizing the full Hamiltonian of the problem considering the coupling between relative and center-of-mass motion up to the fourth lattice band (see Supplemental Material of Ref. [31]). From the fit, in which only the spin-triplet scattering length $a_{e g}^{+}$is left as a free parameter, we estimate $a_{e g}^{+}=1894(18) a_{0}$, where $a_{0}$ is the Bohr radius, a value in agreement with the one determined in Ref. [29].

In a subsequent experiment we demonstrate the capability to drive a coherent molecule photoassociation process $[27,58]$. In this experiment, the clock-laser frequency is kept constant at the photoassociation peak value and the sample of atoms confined in the 3D lattice is probed with clock-laser pulses of increasing duration. Figure 4 reports a typical oscillation at a field $B=150 \mathrm{G}$ and a 3D lattice depth $s_{f}=30$ between pairs of free $|g\rangle-|g\rangle$ atoms and orbital $|g ; e\rangle$ bound states in doubly occupied lattice sites, obtained by detecting the number of $|e\rangle$-state atoms with the same technique used to measure the spectra of Fig. 3. The points are the experimental data and the solid line is the result of a fit to the data with a damped sinusoidal function. Several cycles of photoassociation and photodissociation can be observed with a decay time constant of 7.5(1.6) ms, and the ratio between the frequency $\Omega$ of this oscillation and its single-particle counterpart $\Omega_{0}$ driven with the same clock-laser intensity is a direct measurement of the FranckCondon factor, which is $\mathcal{F}=\Omega / \Omega_{0}=0.81(6)$ at this magnetic-field value. The possibility to coherently excite

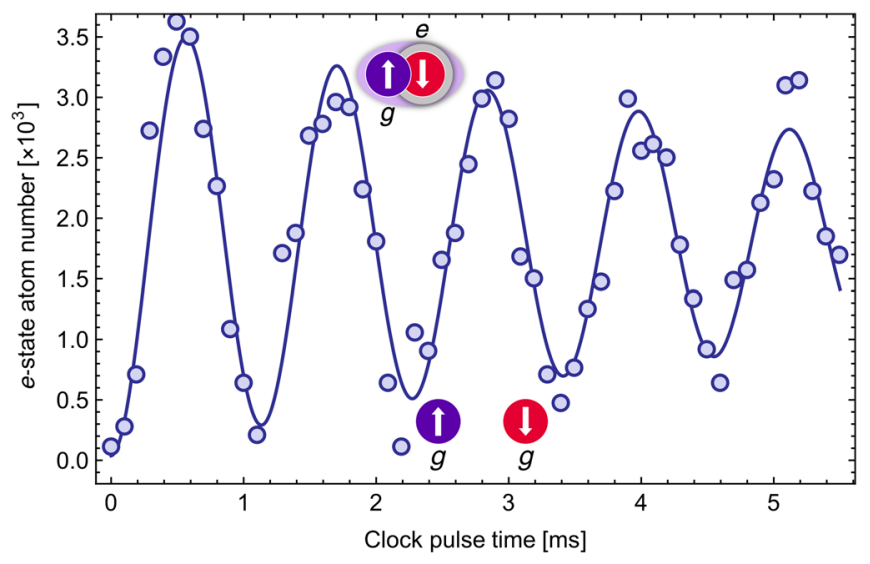

FIG. 4. Rabi oscillation on a $|g\rangle-|e\rangle$ photoassociation peak performed at a magnetic field $B=150 \mathrm{G}$ and 3D lattice depth $s_{f}=30$. The points are the experimental data and the solid curve is a fit with a damped sinusoidal function with a decay time constant of 7.5(1.6) ms. Several periods of photoassociation and photodissociation cycles can be observed. No error bars are present since every point is the result of a single acquisition. pairs of atoms to the bound state is a remarkable feature, which in our experiment is fundamental to maximize the number of orbital molecules in the sample (a goal that is achieved by shining a $\pi$ pulse of clock-laser light at the photoassociation frequency), but is in general interesting for the implementation of new experimental protocols involving the coherent manipulation of two-particle states, e.g., for high-precision spectroscopy [24].

\section{COHERENT SPIN MANIPULATION OF ORBITAL MOLECULES}

In this section, we show how orbital $|g ; e\rangle$ molecules can be coherently manipulated and detected by exploiting Raman transitions acting on the ground-state nuclear-spin d.o.f. of the molecular state. We start by preparing a sample of ${ }^{173} \mathrm{Yb}$ atoms in the $m= \pm 5 / 2$ states in a 3D lattice as reported in the previous section. Atoms in doubly occupied sites are converted into orbital molecules by performing a clock-laser $\pi$ pulse resonant with the photoassociation frequency, so that the sample consists of a 3D array of $|g,+5 / 2 ; e,-5 / 2\rangle$ orbital molecules and individual atoms in either the $m=-5 / 2$ or $m=+5 / 2$ state. We then perform Raman spectroscopy on the sample with a pair of copropagating beams with frequencies $f$ and $f+\Delta f$ detuned by $1756 \mathrm{MHz}$ from the $\left|{ }^{1} S_{0}(F=5 / 2)\right\rangle \rightarrow$ $\left|{ }^{3} P_{1}(F=7 / 2)\right\rangle$ intercombination line $\left(\sim 10^{4}\right.$ times the transition natural linewidth $\Gamma \simeq 180 \mathrm{kHz}$ ), inducing transitions between different nuclear-spin states of the groundstate manifold. The polarization of the two Raman beams is an equal superposition of $\sigma^{+}$and $\sigma^{-}$, thus inducing two possible two-photon processes, namely, $|-5 / 2\rangle \rightarrow|-1 / 2\rangle$ and $|+5 / 2\rangle \rightarrow|+1 / 2\rangle$ transitions [see Fig. 5(a)].

Figure 5(b) reports a typical Raman spectrum at a magnetic field of $B=88 \mathrm{G}$ and a 3D lattice depth of $s_{f}=15$. In order to perform a zero-background measurement, the stretched states $m= \pm 5 / 2$ are selectively blasted with pulses resonant with the magnetically sensitive $\left|{ }^{1} S_{0}\right\rangle \rightarrow\left|{ }^{3} P_{1}\right\rangle$ transition during the time of flight. As a result, only atoms having flipped their spin to other nuclearspin states as a consequence of a Raman process are detected. This detection technique is affected by a small background of a few hundred atoms in the $m \neq \pm 5 / 2$ states as a consequence of imperfections in the optical pumping preparation procedure. In the lower range of two-photon detuning $\Delta f$ between 36 and $38 \mathrm{kHz}$, the spectrum shows two peaks which can be ascribed to the transition of individual $m= \pm 5 / 2$ atoms in singly occupied sites to the $m= \pm 1 / 2$ states. Such processes occur when $\Delta f=\Delta_{\text {at }}$, where $\Delta_{\text {at }}=\Delta m B \times 207 \mathrm{~Hz} / \mathrm{G}$ is the splitting induced by the magnetic field $B$ [see Fig. 5(a)]. While the two transitions should in principle be degenerate, a differential spin-dependent light shift on the nuclear-spin states induced by the Raman light [59] lifts the degeneracy between them. In particular, the beam frequencies and 

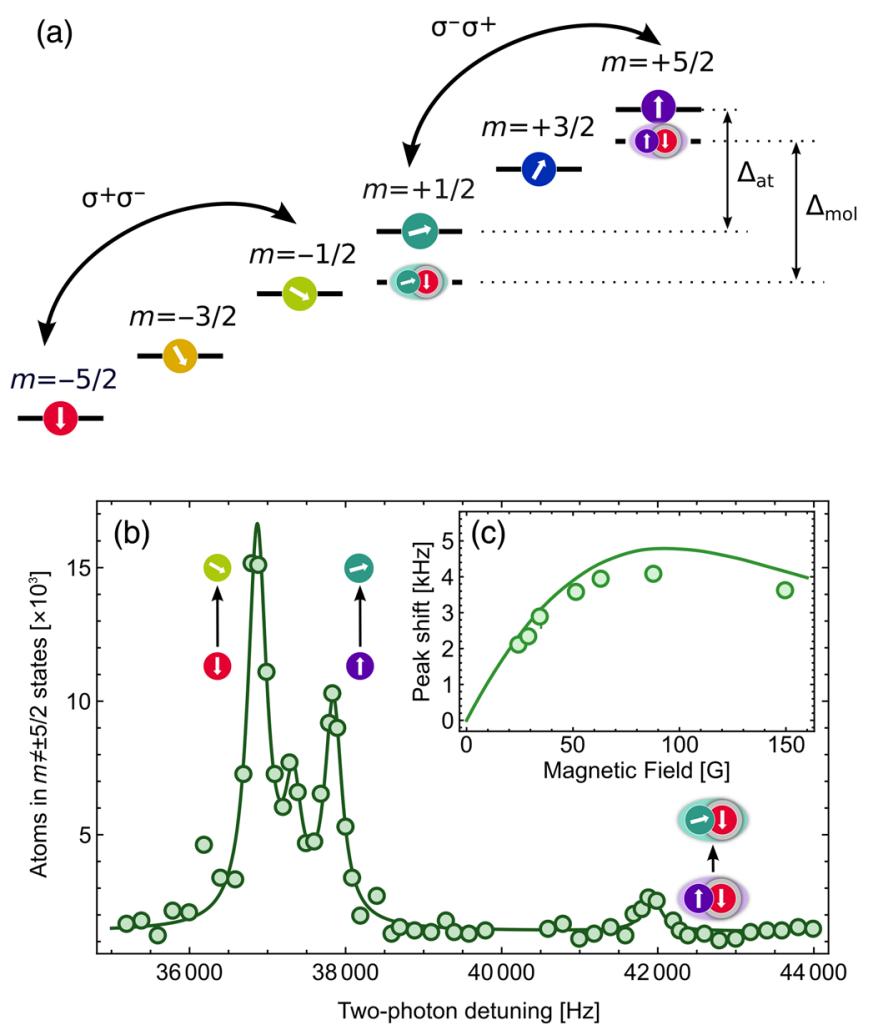

FIG. 5. (a) Scheme of the two-photon Raman transitions connecting different nuclear-spin states. The energy $\Delta_{\text {at }}$ corresponding to a Raman spin flip of individual atoms differs from the energy $\Delta_{\text {mol }}$ needed to flip the spin of the ground-state atom in the orbital molecule (because of the different binding energies for different spin states). (b) Raman spectroscopy of a sample containing individual atoms with $m= \pm 5 / 2$ and orbital molecules at $B=88 \mathrm{G}$ and $s_{f}=15$. The peaks at lower frequency correspond to the spin flipping of individual atoms while the small peak near $42 \mathrm{kHz}$ corresponds to a bound-to-bound transition between orbital molecules made of different spin mixtures. (c) Shift of the bound-to-bound transition peak measured as a function of the magnetic field. The solid line is the binding energy difference between the initial and final molecular states calculated from our model (see Sec. III).

polarizations employed in the experiment result in the $|-5 / 2\rangle \rightarrow|-1 / 2\rangle$ and $|+5 / 2\rangle \rightarrow|+1 / 2\rangle$ transitions being located at lower and higher $\Delta f$, respectively. The different height of the two peaks can be ascribed to the fact that part of the $m=-5 / 2$ particles in the initially balanced sample of ground-state atoms is excited to the $|e\rangle$ state during the molecule creation process. The third smaller peak between the two single-particle transitions can instead be ascribed to resonant four-photon Raman coupling connecting the $m= \pm 5 / 2$ states to $m=\mp 3 / 2$ states.

The most relevant feature of the spectrum in Fig. 5(b) is the small peak located at higher $\Delta f$ around $42 \mathrm{kHz}$. This resonance is connected to a process involving the orbital molecules of the sample, since it disappears when molecules are not present in the sample. We ascribe this peak to a bound-to-bound transition process in which the Raman beams act on the ground-state component of the molecules flipping its spin and transforming a $\mid g,+5 / 2$; $e,-5 / 2\rangle$ molecule into a $|g,+1 / 2 ; e,-5 / 2\rangle$ molecule. The position of this peak is shifted with respect to the single-particle peak(s) due to the different binding energy between the initial and final bound state as a consequence of the binding energy scaling described in Sec. II. Since the resonance for $\Delta m=3$ is located at a higher field than the $\Delta m=5$ case, the final molecule is more deeply bound than the initial one, so the bound-to-bound Raman transition occurs at a higher two-photon detuning than the single-particle case [see Fig. 5(a)], in particular at $\Delta f=\Delta_{\text {mol }}=\Delta_{\text {at }}+\Delta E_{b}$, where $\Delta E_{b}$ is the binding energy difference between the initial and final molecule and $\Delta_{\text {at }}$ is position of the peak corresponding to the $|+5 / 2\rangle \rightarrow|+1 / 2\rangle$ transition.

Furthermore, we measured the shift of the bound-tobound Raman transition peak as a function of the magnetic field at a lattice depth $s=15$. The results of these measurements are reported by the points in Fig. 5(c). The experimental data are compared to the solid line that represents the theoretical prediction of the binding energy difference between two molecules with $\Delta m=5$ and $\Delta m=3$ calculated with our model (see Sec. III). There is a good agreement between the data and the theory, with some deviations at higher magnetic-field values.

Similarly to the what we observed for the photoassociation process with the clock laser, this bound-to-bound Raman transition can be driven coherently, as shown in Fig. 6 where we report a Rabi oscillation performed at a field of $B=150 \mathrm{G}$ and a 3D lattice depth $s_{f}=30$ with a decay time constant of 12(3) ms. Also in this case it should be noted that this oscillation frequency is fully compatible with that of its single-particle counterpart, showing a Frank-Condon factor which approaches unity.

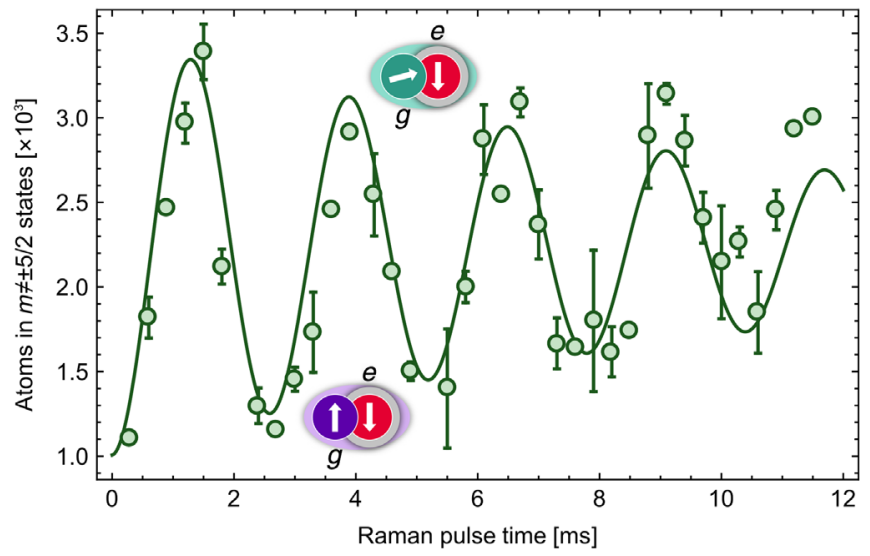

FIG. 6. Rabi oscillation on the bound-to-bound Raman transition peak performed at $B=150 \mathrm{G}$ and a $3 \mathrm{D}$ lattice depth $s_{f}=30$. The Raman light coherently flips the ground-state nuclear-spin component of the molecules between the $m=+5 / 2$ state and $m=+1 / 2$ state, with a decay time constant of $12(3) \mathrm{ms}$. 
We exploit this property to perform a "triple-pulse" experiment in which we combine clock-laser photoassociation and Raman manipulation with the goal to verify our capability to manipulate both the orbital $(|g\rangle,|e\rangle)$ and nuclear-spin d.o.f. in a coherent way and to further validate the attribution of the bound-to-bound transition peak in Fig. 5(b). Initially, a sample of $m= \pm 5 / 2$ atoms is prepared in a 3D lattice and orbital molecules are created in doubly occupied sites with a clock-laser $\pi$ pulse at a fixed frequency resonant with the photoassociation transition. We then perform a Raman $\pi$ pulse at the bound-tobound transition frequency so that the $|g,+5 / 2 ; e,-5 / 2\rangle$ molecules are converted into $|g,+1 / 2 ; e,-5 / 2\rangle$ molecules and finally a second clock-laser $\pi$ pulse is shone on the sample. Differently from the first clock-laser photoassociation pulse, the frequency of this second clock-laser pulse is scanned in order to perform photodissociation spectroscopy. As in the photoassociation spectroscopy experiment of Fig. 3, we detect the number of atoms in the $|e\rangle$ state. The results of this experiment for $B=150 \mathrm{G}$ and $s_{f}=30$ are represented by the green points in Fig. 7, while the solid green line is a fit to the data with a double Lorentzian function. Depending on the frequency of the clock laser during the second $\pi$ pulse, different outcomes are possible. If the second clock-laser pulse is not resonant with any transition [see (a) in Fig. 7], a "background" number of approximately $5 \times 10^{3}|e\rangle$-state atoms is detected, corresponding to the $|e\rangle$-state atoms forming the orbital molecules produced with the first clock-laser photoassociation $\pi$ pulse. In case the second clock pulse is resonant with the excitation of individual $m=-5 / 2$ atoms in singly occupied sites from $|g\rangle$ to $|e\rangle$, we detect an increase of $|e\rangle$-state atoms [see (b) in Fig. 7], as a consequence of additional individual $|e\rangle$ atoms summing to the preexisting $|e\rangle$ atoms forming the orbital molecules created with the first photoassociation pulse. In analogy with the clock photoassociation spectrum of Fig. 3, the position of this peak is taken as the zero-frequency reference. Finally, when the second clock-laser pulse is resonant with the molecular peak, the orbital molecules are dissociated to a pair of $|g\rangle$-state atoms and a depletion of $|e\rangle$-state atoms is detected [see (c) in Fig. 7]. At the center of the resonance, this depletion is noticeably high, with less than $10^{3}$ atoms that remain in the excited state corresponding to $<20 \%$ of the initial number of molecules. For comparison, the blue points and fit in Fig. 7 are the result of the same experiment performed without shining the Raman $\pi$ pulse on the bound-to-bound transition, so that orbital molecules are associated with the first clock-laser $\pi$ pulse followed immediately by the second clock-laser $\pi$ pulse with varying frequency. We detect the same number of background $|e\rangle$-state atoms and a similar dissociation peak, which is located at higher frequency [see (d) in Fig. 7] with respect to the case in which $\Delta m=3$ molecules are created by the Raman pulse because of the smaller binding energy of the molecules

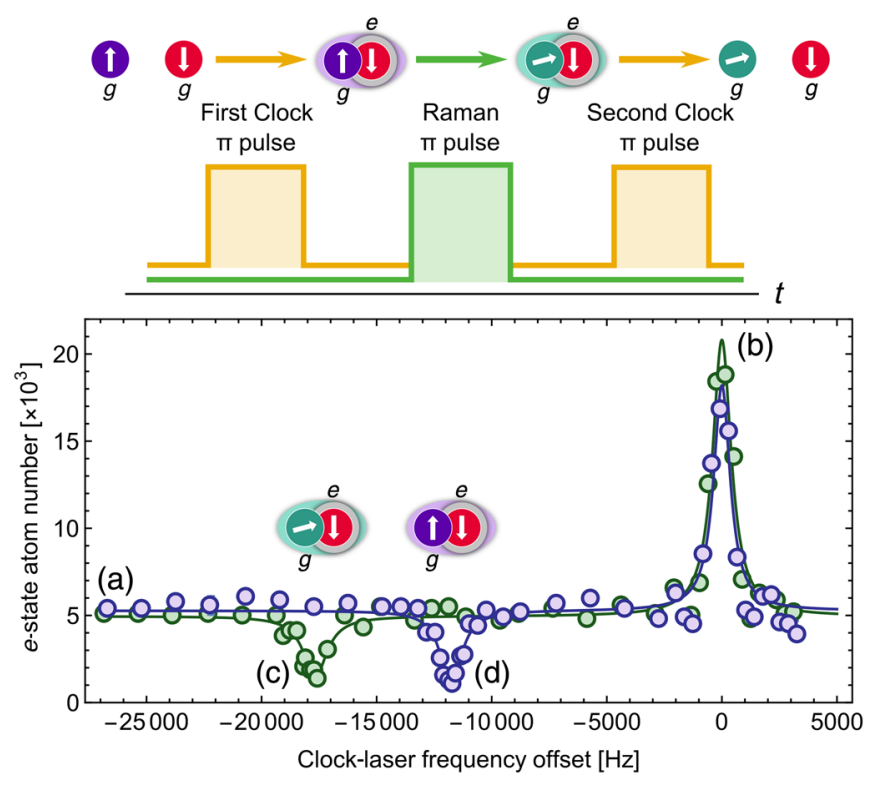

FIG. 7. Clock-laser spectroscopy of a sample with individual atoms and orbital molecules produced with a preliminary clocklaser $\pi$ pulse. The excitation of individual atoms is detected as an increase in the number of $|e\rangle$-state atoms (b) with respect to the background (a) and serves a zero-frequency reference. The dissociation of orbital molecules is instead detected as a decrease of $|e\rangle$-state atoms (c). The blue data correspond to an experiment in which the sample is probed immediately after the production of orbital molecules, while the green data correspond to an experiment in which the nuclear-spin state of ground-state component of the molecules has been flipped from $+5 / 2$ to $+1 / 2$ with a bound-to-bound Raman transition (see text for details). The frequency difference between the two dissociation peaks corresponds to the energy difference between the bound states of the two different mixtures of spin components at $150 \mathrm{G}$ and a $s=30$ lattice depth (see Fig. 2). Both experiments are performed using a sequence of coherent $\pi$ pulses, as can be seen by the nearly full depletion of the photodissociation peaks.

with $\Delta m=5$. The frequency difference between the dissociation peaks of the two spectra in Fig. 7 corresponds to the two-photon detuning difference between the Raman single-particle and bound-to-bound transition peaks of the analogous Raman spectrum of Fig. 5(b) performed at $B=150 \mathrm{G}$, definitely demonstrating the bound-to-bound nature of the Raman process.

\section{LIFETIME OF THE MOLECULAR SAMPLE}

In this section, we investigate the lifetime of orbital molecules in different lattice configurations. The simple detection of $|e\rangle$-state atoms is not suitable for this kind of measurement, as it could be possible for the orbital molecules to dissociate spontaneously into pairs of independent $|g\rangle$ and $|e\rangle$ atoms during the hold time, leading to an overestimation of the real number of molecules. The Raman spectroscopy technique discussed in the previous section is a more suitable method, as the presence of a 
bound-to-bound transition peak in the Raman spectrum is an unambiguous signature of orbital molecules in the sample. In particular, their number can be measured by counting the number of $|g\rangle$ atoms in $m \neq \pm 5 / 2$ states after a Raman pulse at the bound-to-bound transition frequency.

In a first experiment, we study the lifetime of isolated molecules confined in a 3D optical lattice. This is done by photoassociating molecules with a clock-laser $\pi$ pulse in a 3D lattice of depth $s_{f}$ and at a magnetic field $B$, as in the previous experiments. The sample is then held in the 3D lattice for a variable hold time, and a Raman $\pi$ pulse resonant with the bound-to-bound two-photon transition is performed after the hold time in order to detect the number of molecules. The blue data in Fig. 8 correspond to the result of this measurement as a function of the hold time in a $s_{f}=153 \mathrm{D}$ lattice performed at $B=25 \mathrm{G}$. We fit the data with an exponential curve obtaining a lifetime of the isolated molecules of $0.35(7) \mathrm{s}$, a remarkably long value despite one of the atoms forming the molecule being in a highly excited state. The main limiting factor to the lifetime could be identified as inelastic losses due to collisions after a tunneling process to an occupied neighboring site (the tunneling rate is of the order of $10 \mathrm{~Hz}$ ). As mentioned before, we note that a small background of a few hundred atoms in the $m \neq \pm 5 / 2$ states is present as a consequence of imperfections in the initial preparation of the sample.

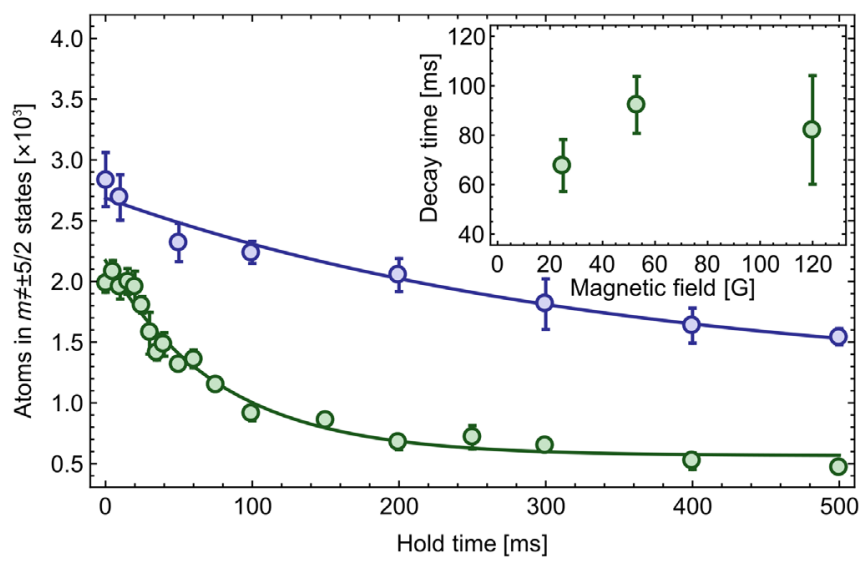

FIG. 8. Lifetime measurements of orbital molecules in different optical lattice configurations. Blue: Lifetime of isolated orbital molecules in a $s_{f}=153 \mathrm{D}$ lattice. An exponential fit to the data results in a decay time constant of $0.35(0.07)$ s. Green: Lifetime of interacting molecules in an array of pancakes. In this second scenario, molecules are free to interact with other atoms and molecules of the sample, leading to a shorter lifetime with respect to the 3D lattice case. The nonzero background of a few hundred atoms in the $m \neq \pm 5 / 2$ states can be ascribed to an imperfect preparation of the initial sample. Inset: Measured lifetime of orbital molecules in pancakes for different values of magnetic field across the orbital Feshbach resonance. The lifetime values are compatible within the error bars, and no evident trend is observable.
In a second experiment, we use this technique to carry out a first study of orbital molecule lifetime in a many-body environment. After the clock photoassociation of molecules in the 3D lattice, the horizontal lattice beams are turned off with a 1-ms-long linear ramp to obtain a vertical stack of pancakes with a radial trapping frequency of approximately $2 \pi \times 20 \mathrm{~Hz}$ and a strong axial confinement of $2 \pi \times 15 \mathrm{kHz}$, corresponding to an optical lattice depth of $s=15$ along the vertical direction. The sample is held in this pancake configuration for a variable hold time, then the horizontal lattices are turned on again with 1-mslong linear ramps and a Raman $\pi$ pulse is performed to detect the number of remaining molecules, as in the previous experiment. The results of such an experiment performed at a field of $25 \mathrm{G}$ are reported by the green data in Fig. 8, where it is compared to the lifetime of isolated orbital molecules in the 3D lattice. In the pancakes, the molecules are free to interact with both other molecules and single ground-state atoms in the $m= \pm 5 / 2$ states. The decay rate of the molecules could than be described by a rate equation $\dot{n}_{m}=-\beta_{m a} n_{m} n_{a}-\beta_{m m} n_{m}^{2}$, where $n_{m}$ and $n_{a}$ are the density of molecules and individual atoms, respectively, and $\beta_{m a}$ and $\beta_{m m}$ are the loss rate coefficients of the molecule-atom and molecule-molecule loss processes, respectively. In our experimental conditions, the typical number of molecules (a few thousand) is much smaller than the typical number of atoms (typically of the order of $50 \times 10^{3}$ ), so it is reasonable to assume that the dominant loss mechanism is represented by molecule-atom collisions. In this case, the second term on the right-hand side of the previous rate equation can be neglected, and the model reduces to a simpler rate equation $\dot{n}_{m}=-\beta_{m a} n_{m} n_{a}$, which is solved by a single exponential curve $n_{m}=$ $e^{-\beta_{m a} n_{a} t}=e^{-\gamma_{m a} t}$. We fit the green data of Fig. 8 with an exponential function obtaining a lifetime of $1 / \gamma_{m a}=$ $77(8) \mathrm{ms}$. In order to quantify the loss rate coefficient related to this process, we use a simplified model to calculate the average density in every pancake starting from our initial sample with $N$ atoms and $T=0.25 T_{F}$, similarly to the model used to calculate the density of bosonic ${ }^{174} \mathrm{Yb}$ in Ref. [55]. We then average over all the pancakes to obtain a mean density value of $n_{\text {at }}=$ $6.3(3.1) \times 10^{12} \mathrm{~cm}^{-3}$, to which we attribute a conservative error due to several assumptions in our theoretical model. From this value we can determine a loss rate coefficient $\beta_{m a}=\gamma_{m a} n_{a}=2.1(1.2) \times 10^{-12} \mathrm{~cm}^{3} \mathrm{~s}^{-1}$.

This method allowed us to measure the molecule's lifetime in the sample for different values of magnetic field all across the free-space orbital Feshbach resonance. The results of these measurements are reported in the inset of Fig. 8. Each point is the average of two independent experiments performed in different days and the error bar is the average of the fit errors of the individual experiments. Noticeably, there is not a clearly visible trend and the measured lifetimes are compatible within the error bars, 
independently of the magnetic field being set below or above or near resonance. This is in general not true for Feshbach molecules of fermionic alkali atoms, which show faster losses on the BEC side of the resonance and a longer lifetime near resonance arising from the Pauli exclusion which suppresses collisions between the fermionic constituents of the weakly bound dimers. This fact is a further suggestion that our lifetime measurements are dominated by molecule-atom losses, which are not protected by the Pauli exclusion principle due to the presence of atoms in a "third" state (the $|g,-5 / 2\rangle$ atoms in the singly occupied sites of the initial 3D lattice) different from both the states of atoms forming the $|g,+5 / 2 ; e,-5 / 2\rangle$ orbital molecule. A more detailed investigation of the multiple interaction mechanisms, especially of molecule-molecule scattering, would require the creation of pure samples of orbital molecules, a very difficult task in $\mathrm{Yb}$ samples due to the small binding energy of the molecules, which makes it impossible to selectively address and blast from the sample single atoms exploiting "nonclock" transitions. Nevertheless, the measured lifetimes of the order of $100 \mathrm{~ms}$ are a promising starting point for future studies of the BEC-BCS crossover in this unexplored regime, as it represents a lower limit to the lifetime that could be obtained in pure molecular samples.

\section{CONCLUSIONS}

In this paper we demonstrate the possibility to produce and coherently manipulate Feshbach molecules made by two-electron atoms in different electronic "clock" states ${ }^{1} S_{0}$ and ${ }^{3} P_{0}$ and provide a first investigation of the molecule lifetime in a many-body setting. We show how these molecules can be produced in a three-dimensional optical lattice by means of direct optical photoassociation with clock-laser light and demonstrate that this photoassociation process can be driven coherently, leading to several cycles of association and dissociation. Noticeably, the achievable Rabi frequencies are comparable to that of the excitation of a single-atom clock transition under the same experimental conditions, highlighting a favorable Frank-Condon factor. This opens new possibilities towards the realization of molecular optical clocks [24], as well as for the exploration of new approaches for the control of interactions in twoelectron atoms with optical Feshbach resonances [60-62].

We also demonstrate that the molecular internal d.o.f. can be controlled exploiting Raman transitions that swap between different nuclear-spin states of the ${ }^{1} S_{0}$ atom forming the molecule. We show also that this process can be driven coherently, allowing us to cycle between molecular states with different ground-state components and providing a very powerful manipulation tool, which could also be exploited for the optical tuning of an optical Feshbach resonance [63]. Moreover, we show that this manipulation scheme can also be used as a detection tool for orbital molecules, which are characterized by an extremely shallow binding energy that causes the usual detection methods of Feshbach molecules of alkaline atoms to be unusable. This allowed us to perform a first investigation of orbital molecules in a many-body environment, where we measured lifetimes approaching $100 \mathrm{~ms}$. This value, which is already of the same order of the typical experimental timescales, is particularly promising since it appears to be mainly limited by the presence of a large number of unpaired atoms in the sample, and is therefore a lower limit to the lifetime that could be achieved in a pure sample of molecules. In future work, the possible development of a "purification" method and improved molecule production techniques could allow for the creation of pure and larger samples of molecules leading to further and more detailed experiments to characterize the different scattering processes of orbital molecules. potentially opening the way to the investigation of the BEC to BCS crossover in atoms with two internal d.o.f.

\section{ACKNOWLEDGMENTS}

We are very grateful to H. Zhai, P. Zhang, R. Zhang, and Y. Cheng for the numerous inspiring discussions and for suggesting that we perform Raman measurements on orbital molecules. We also acknowledge insightful discussions within the LENS QuantumGases group, in particular, with G. Roati, F. Scazza, and M. Zaccanti. We thank D. Calonico (INRIM) for lending us the clock-laser chip, and TOPTICA Photonics AG for their prompt and high-quality technical assistance. We acknowledge financial support from H2020 European Research Council (ERC Consolidator Grant TOPSIM Grant Agreement No. 682629), European QuantERA ERA-NET Cofund in Quantum Technologies (Project QTFLAG Grant Agreement No. 731473), Ministero dell'Istruzione, dell'Università e della Ricerca (MIUR Project FARE TOPSPACE R16SPCCRCW, and MIUR PRIN Project No. 2015C5SEJJ) and Istituto Nazionale di Fisica Nucleare (INFN Project FISh).

[1] L. D. Carr, D. DeMille, R. V. Krems, and J. Ye, Cold and Ultracold Molecules: Science, Technology and Applications, New J. Phys. 11, 055049 (2009).

[2] C. Chin, V. V. Flambaum, and M. G. Kozlov, Ultracold Molecules: New Probes on the Variation of Fundamental Constants, New J. Phys. 11, 055048 (2009).

[3] M. S. Safronova, D. Budker, D. DeMille, D. F. J. Kimball, A. Derevianko, and C. W. Clark, Search for New Physics with Atoms and Molecules, Rev. Mod. Phys. 90, 025008 (2018).

[4] S. A. Moses, J. P. Covey, M. T. Miecnikowski, B. Yan, B. Gadway, J. Ye, and D. S. Jin, Creation of a Low-Entropy Quantum Gas of Polar Molecules in an Optical Lattice, Science 350, 659 (2015).

[5] L. Reichsllner, A. Schindewolf, T. Takekoshi, R. Grimm, and H.-C. Nägerl, Quantum Engineering of a Low-Entropy 
Gas of Heteronuclear Bosonic Molecules in an Optical Lattice, Phys. Rev. Lett. 118, 073201 (2017).

[6] J. W. Park, S. A. Will, and M.W. Zwierlein, Ultracold Dipolar Gas of Fermionic ${ }^{23} \mathrm{Na}^{40} \mathrm{~K}$ Molecules in Their Absolute Ground State, Phys. Rev. Lett. 114, 205302 (2015).

[7] P. K. Molony, P. D. Gregory, Z. Ji, B. Lu, M. P. Kppinger, C. R. Le Sueur, C. L. Blackley, J. M. Hutson, and S. L. Cornish, Creation of Ultracold ${ }^{87} \mathrm{Rb}^{133} \mathrm{Cs}$ Molecules in the Rovibrational Ground State, Phys. Rev. Lett. 113, 255301 (2014).

[8] M. Guo, B. Zhu, B. Lu, X. Ye, F. Wang, R. Vexiau, N. Bouloufa-Maafa, G. Qumner, O. Dulieu, and D. Wang, Creation of an Ultracold Gas of Ground-State Dipolar ${ }^{23} \mathrm{Na}^{87} \mathrm{Rb}$ Molecules, Phys. Rev. Lett. 116, 205303 (2016).

[9] F. Seeßelberg, N. Buchheim, Z.-K. Lu, T. Schneider, X.-Y. Luo, E. Tiemann, I. Bloch, and C. Gohle, Modeling the Adiabatic Creation of Ultracold Polar ${ }^{23} \mathrm{Na}^{40} \mathrm{~K}$ Molecules, Phys. Rev. A 97, 013405 (2018).

[10] T. M. Rvachov, H. Son, A. T. Sommer, S. Ebadi, J. J. Park, M. W. Zwierlein, W. Ketterle, and A. O. Jamison, LongLived Ultracold Molecules with Electric and Magnetic Dipole Moments, Phys. Rev. Lett. 119, 143001 (2017).

[11] L. De Marco, G. Valtolina, K. Matsuda, W. G. Tobias, J. P. Covey, and J. Ye, A Fermi Degenerate Gas of Polar Molecules, arXiv:1808.00028.

[12] A. Micheli, G. K. Brennen, and P. Zoller, A Toolbox for Lattice-Spin Models with Polar Molecules, Nat. Phys. 2, 341 (2006).

[13] B. Yan, S. A. Moses, B. Gadway, J. P. Covey, K. R. A. Hazzard, A. M. Rey, D. S. Jin, and J. Ye, Observation of Dipolar Spin-Exchange Interactions with Lattice-Confined Polar Molecules, Nature (London) 501, 521 (2013).

[14] S. Ospelkaus, K.-K. Ni, D. Wang, M. H. G. de Miranda, B. Neyenhuis, G. Qumner, P. S. Julienne, J. L. Bohn, D. S. Jin, and J. Ye, Quantum-State Controlled Chemical Reactions of Ultracold Potassium-Rubidium Molecules, Science 327, 853 (2010).

[15] M. McDonald, B. H. McGuyer, F. Apfelbeck, C.-H. Lee, I. Majewska, R. Moszynski, and T. Zelevinsky, Photodissociation of Ultracold Diatomic Strontium Molecules with Quantum State Control, Nature (London) 535, 122 (2016).

[16] M. Zeppenfeld, B. G. U. Englert, R. Glöckner, A. Prehn, M. Mielenz, C. Sommer, L. D. van Buuren, M. Motsch, and G. Rempe, Sisyphus Cooling of Electrically Trapped Polyatomic Molecules, Nature (London) 491, 570 (2012).

[17] J. F. Barry, D. J. McCarron, E. B. Norrgard, M. H. Steinecker, and D. DeMille, Magneto-optical Trapping of a Diatomic Molecule, Nature (London) 512, 286 (2014).

[18] S. Truppe, H. J. Williams, M. Hambach, L. Caldwell, N. J. Fitch, E. A. Hinds, B. E. Sauer, and M. R. Tarbutt, Molecules Cooled Below the Doppler Limit, Nat. Phys. 13, 1173 (2017).

[19] B. Hemmerling, E. Chae, A. Ravi, L. Anderegg, G. K. Drayna, N. R. Hutzler, A. L. Collopy, J. Ye, W. Ketterle, and J. M. Doyle, Laser Slowing of CaF Molecules to Near the Capture Velocity of a Molecular MOT, J. Phys. B 49, 174001 (2016).

[20] C. Chin, R. Grimm, P. Julienne, and E. Tiesinga, Feshbach Resonances in Ultracold Gases, Rev. Mod. Phys. 82, 1225 (2010).
[21] B. J. Bloom, T. L. Nicholson, J. R. Williams, S. L. Campbell, M. Bishof, X. Zhang, W. Zhang, S. L. Bromley, and J. Ye, An Optical Lattice Clock with Accuracy and Stability at the $10^{18}$ Level, Nature (London) 506, 71 (2014).

[22] I. Ushijima, M. Takamoto, M. Das, T. Ohkubo, and H. Katori, Cryogenic Optical Lattice Clocks, Nat. Photonics 9, 185 (2015).

[23] W. F. McGrew, X. Zhang, R. J. Fasano, S. A. Schäffer, K. Beloy, D. Nicolodi, R. C. Brown, N. Hinkley, G. Milani, M. Schioppo, T. H. Yoon, and A. D. Ludlow, Atomic Clock Performance Beyond the Geodetic Limit, Nature (London) 564, 87 (2018).

[24] M. Borkowski, Optical Lattice Clocks with Weakly Bound Molecules, Phys. Rev. Lett. 120, 083202 (2018).

[25] B. H. McGuyer, M. McDonald, G. Z. Iwata, M. G. Tarallo, W. Skomorowski, R. Moszynski, and T. Zelevinsky, Precise Study of Asymptotic Physics with Subradiant Ultracold Molecules, Nat. Phys. 11, 32 (2015).

[26] B. H. McGuyer, M. McDonald, G. Z. Iwata, M. G. Tarallo, A. T. Grier, F. Apfelbeck, and T. Zelevinsky, High-Precision Spectroscopy of Ultracold Molecules in an Optical Lattice, New J. Phys. 17, 055004 (2015).

[27] S. Taie, S. Watanabe, T. Ichinose, and Y. Takahashi, Feshbach-Resonance-Enhanced Coherent Atom-Molecule Conversion with Ultranarrow Photoassociation Resonance, Phys. Rev. Lett. 116, 043202 (2016).

[28] Y. Takasu, Y. Fukushima, Y. Nakamura, and Y. Takahashi, Magnetoassociation of a Feshbach Molecule and SpinOrbit Interaction Between the Ground and Electronically Excited States, Phys. Rev. A 96, 023602 (2017).

[29] M. Höfer, L. Riegger, F. Scazza, C. Hofrichter, D. R. Fernandes, M. M. Parish, J. Levinsen, I. Bloch, and S. Fölling, Observation of an Orbital Interaction-Induced Feshbach Resonance in ${ }^{173} \mathrm{Yb}$, Phys. Rev. Lett. 115, 265302 (2015).

[30] R. Zhang, Y. Cheng, H. Zhai, and P. Zhang, Orbital Feshbach Resonance in Alkali-Earth Atoms, Phys. Rev. Lett. 115, 135301 (2015).

[31] G. Cappellini, M. Mancini, G. Pagano, P. Lombardi, L. Livi, M. S. de Cumis, P. Cancio, M. Pizzocaro, D. Calonico, F. Levi, C. Sias, J. Catani, M. Inguscio, and L. Fallani, Direct Observation of Coherent Interorbital Spin-Exchange Dynamics, Phys. Rev. Lett. 113, 120402 (2014).

[32] F. Scazza, C. Hofrichter, M. Höfer, P. C. De Groot, I. Bloch, and S. Fölling, Observation of Two-Orbital Spin-Exchange Interactions with Ultracold SU(N)-Symmetric Fermions, Nat. Phys. 10, 779 (2014).

[33] G. Pagano, M. Mancini, G. Cappellini, L. Livi, C. Sias, J. Catani, M. Inguscio, and L. Fallani, Strongly Interacting Gas of Two-Electron Fermions at an Orbital Feshbach Resonance, Phys. Rev. Lett. 115, 265301 (2015).

[34] Y. Cheng, R. Zhang, and P. Zhang, Quantum Defect Theory for the Orbital Feshbach Resonance, Phys. Rev. A 95, 013624 (2017).

[35] L. He, J. Wang, S.-G. Peng, X.-J. Liu, and H. Hu, Strongly Correlated Fermi Superfluid Near an Orbital Feshbach Resonance: Stability, Equation of State, and Leggett Mode, Phys. Rev. A 94, 043624 (2016). 
[36] Y.-C. Zhang, S. Ding, and S. Zhang, Collective Modes in a Two-Band Superfluid of Ultracold Alkaline-Earth-Metal Atoms Close to an Orbital Feshbach Resonance, Phys. Rev. A 95, 041603(R) (2017).

[37] J. Xu, R. Zhang, Y. Cheng, P. Zhang, R. Qi, and H. Zhai, Reaching a Fermi-Superfluid State Near an Orbital Feshbach Resonance, Phys. Rev. A 94, 033609 (2016).

[38] L. F. Livi, G. Cappellini, M. Diem, L. Franchi, C. Clivati, M. Frittelli, F. Levi, D. Calonico, J. Catani, M. Inguscio, and L. Fallani, Synthetic Dimensions and Spin-Orbit Coupling with an Optical Clock Transition, Phys. Rev. Lett. 117, 220401 (2016).

[39] S. Kolkowitz, S. L. Bromley, T. Bothwell, M. L. Wall, G. E. Marti, A. P. Koller, X. Zhang, A. M. Rey, and J. Ye, SpinOrbit-Coupled Fermions in an Optical Lattice Clock, Nature (London) 542, 66 (2017).

[40] Y. Takasu, Y. Fukushima, Y. Nakamura, and Y. Takahashi, Magnetoassociation of a Feshbach Molecule and SpinOrbit Interaction Between the Ground and Electronically Excited States, Phys. Rev. A 96, 023602 (2017).

[41] X.-J. Liu, L. Jiang, H. Pu, and H. Hu, Probing Majorana Fermions in Spin-Orbit-Coupled Atomic Fermi Gases, Phys. Rev. A 85, 021603(R) (2012).

[42] X.-J. Liu and H. Hu, Topological Superfluid in OneDimensional Spin-Orbit-Coupled Atomic Fermi Gases, Phys. Rev. A 85, 033622 (2012).

[43] H. Zhai, Degenerate Quantum Gases with SpinOrbit Coupling: A Review, Rep. Prog. Phys. 78, 026001 (2015).

[44] F. Iemini, L. Mazza, L. Fallani, P. Zoller, R. Fazio, and M. Dalmonte, Majorana Quasiparticles Protected by $\mathbb{Z}_{2}$ Angular Momentum Conservation, Phys. Rev. Lett. 118, 200404 (2017).

[45] J. Cubizolles, T. Bourdel, S. J. J. M. F. Kokkelmans, G. V. Shlyapnikov, and C. Salomon, Production of Long-Lived Ultracold $\mathrm{Li}_{2}$ Molecules from a Fermi Gas, Phys. Rev. Lett. 91, 240401 (2003).

[46] M. Greiner, C. A. Regal, and D. S. Jin, Emergence of a Molecular Bose-Einstein Condensate from a Fermi Gas, Nature (London) 426, 537 (2003).

[47] S. Jochim, M. Bartenstein, A. Altmeyer, G. Hendl, S. Riedl, C. Chin, J. H. Denschlag, and R. Grimm, Bose-Einstein Condensation of Molecules, Science 302, 2101 (2003).

[48] M. W. Zwierlein, C. A. Stan, C. H. Schunck, S. M. F. Raupach, S. Gupta, Z. Hadzibabic, and W. Ketterle, Observation of Bose-Einstein Condensation of Molecules, Phys. Rev. Lett. 91, 250401 (2003).

[49] A. V. Gorshkov, M. Hermele, V. Gurarie, C. Xu, P. S. Julienne, J. Ye, P. Zoller, E. Demler, M. D. Lukin, and A. M. Rey, Two-Orbital SU(N) Magnetism with Ultracold Alkaline-Earth Atoms, Nat. Phys. 6, 289 (2010).

[50] T. Stöferle, H. Moritz, K. Günter, M. Köhl, and T. Esslinger, Molecules of Fermionic Atoms in an Optical Lattice, Phys. Rev. Lett. 96, 030401 (2006).
[51] G. Thalhammer, K. Winkler, F. Lang, S. Schmid, R. Grimm, and J. H. Denschlag, Long-Lived Feshbach Molecules in a Three-Dimensional Optical Lattice, Phys. Rev. Lett. 96, 050402 (2006).

[52] C. Ospelkaus, S. Ospelkaus, L. Humbert, P. Ernst, K. Sengstock, and K. Bongs, Ultracold Heteronuclear Molecules in a 3D Optical Lattice, Phys. Rev. Lett. 97, 120402 (2006).

[53] G. Cappellini, P. Lombardi, M. Mancini, G. Pagano, M. Pizzocaro, L. Fallani, and J. Catani, A Compact Ultranarrow High-Power Laser System for Experiments with $578 \mathrm{~nm}$ Ytterbium Clock Transition, Rev. Sci. Instrum. 86, 073111 (2015).

[54] S. L. Campbell, R. B. Hutson, G. E. Marti, A. Goban, N. D. Oppong, R. L. McNally, L. Sonderhouse, J. M. Robinson, W. Zhang, B. J. Bloom, and J. Ye, A Fermi-Degenerate Three-Dimensional Optical Lattice Clock, Science 358, 90 (2017).

[55] L. Franchi, L. F. Livi, G. Cappellini, G. Binella, M. Inguscio, J. Catani, and L. Fallani, State-Dependent Interactions in Ultracold ${ }^{174} \mathrm{Yb}$ Probed by Optical Clock Spectroscopy, New J. Phys. 19, 103037 (2017).

[56] M. Kitagawa, K. Enomoto, K. Kasa, Y. Takahashi, R. Ciuryo, P. Naidon, and P. S. Julienne, Two-Color Photoassociation Spectroscopy of Ytterbium Atoms and the Precise Determinations of s-Wave Scattering Lengths, Phys. Rev. A 77, 012719 (2008).

[57] T. Busch, B.-G. Englert, K. Rzaewski, and M. Wilkens, Two Cold Atoms in a Harmonic Trap, Found. Phys. 28, 549 (1998).

[58] Z. Fu, L. Huang, Z. Meng, P. Wang, L. Zhang, S. Zhang, H. Zhai, P. Zhang, and J. Zhang, Production of Feshbach Molecules Induced by Spin-Orbit Coupling in Fermi Gases, Nat. Phys. 10, 110 (2014).

[59] M. Mancini, G. Pagano, G. Cappellini, L. F. Livi, M. Rider, J. Catani, C. Sias, P. Zoller, M. Inguscio, M. Dalmonte, and L. Fallani, Observation of Chiral Edge States with Neutral Fermions in Synthetic Hall Ribbons, Science 349, 1510 (2015).

[60] M. Theis, G. Thalhammer, K. Winkler, M. Hellwig, G. Ruff, R. Grimm, and J.H. Denschlag, Tuning the Scattering Length with an Optically Induced Feshbach Resonance, Phys. Rev. Lett. 93, 123001 (2004).

[61] S. Blatt, T. L. Nicholson, B. J. Bloom, J. R. Williams, J. W. Thomsen, P. S. Julienne, and J. Ye, Measurement of Optical Feshbach Resonances in an Ideal Gas, Phys. Rev. Lett. 107, 073202 (2011).

[62] O. Thomas, C. Lippe, T. Eichert, and H. Ott, Experimental Realization of a Rydberg Optical Feshbach Resonance in a Quantum Many-Body System, Nat. Commun. 9, 2238 (2018).

[63] Z. Han and T.-S. Deng, Dressing the Orbital Feshbach Resonance Using Single-Manifold Raman Scheme, arXiv: 1808.07578. 